\title{
Posterior Intraorbital Foreign Body: Take it or Leave it?
}

\author{
Robert J. Peralta ${ }^{*}, 1,2$, Christopher Zoumalan ${ }^{1,2}$ and Gary J. Lelli Jr. ${ }^{3}$ \\ ${ }^{1}$ Department of Ophthalmology, New York University School of Medicine, USA \\ ${ }^{2}$ Department of Ophthalmology, Manhattan Eye, Ear and Throat Hospital, USA \\ ${ }^{3}$ Weill Cornell Department of Ophthalmology, New York Presbyterian Hospital, USA
}

\begin{abstract}
Intraorbital foreign body (IoFB) presents an interesting therapeutic dilemma when located posterior in the orbit. Factors such as foreign body composition, inflammation, infection, functional deficit, and potential for iatrogenic damage are considered when choosing the appropriate treatment.
\end{abstract}

\section{CASE REPORT}

A 20 year-old male presented to the Bellevue Hospital emergency department one day after suffering a high velocity projectile penetrating injury (BB pellet) to the right orbit. Best corrected visual acuity was 20/40 OD and 20/20 OS. Clinical examination of the right eye revealed an entrance wound $10 \mathrm{~mm}$ inferior to the medial canthus but no exit wound, mild pain and limitation of extraocular movements, mild relative afferent pupillary defect (APD) and complete loss of color vision on Ishihara testing. Computed tomography identified a metallic foreign body deep in the posterior orbit near the orbital apex and right optic nerve (Fig. 1). He was admitted and treated with broad spectrum antibiotics (ancef $1 \mathrm{~g} \mathrm{q} 8 \mathrm{~h}$ ) and intravenous corticosteroids (prednisolone $1 \mathrm{~g}$ loading dose followed by $250 \mathrm{mg}$ q6h for two days). Two days after his injury, visual acuity returned to 20/20 OD with full resolution of motility, relative APD, and color vision deficits. The patient then left the hospital against medical advice and was lost to further follow-up.

\section{DISCUSSION}

There is a paucity of literature regarding the management of posteriorly located IoFBs, owing most likely to the relatively uncommon nature of this phenomenon. A small retrospective study by Finkelstein et al. reviewed 27 consecutive patients over 7 years [1]. All cases involved metallic foreign bodies, the majority of which were BB gun injuries (20 of 27). 13 of the foreign bodies were located in the anterior orbit, 4 epibulbar and 10 posterior. Practically all (94\%, 16 of 17) anterior and epibulbar foreign bodies were surgically removed, whereas only $20 \%$ ( 2 of 10 ) of those located posteriorly were extricated (which occured during ruptured globe repair). Most common associated ocular morbidities included local trauma (subconjunctival hemorrhage, corneal abrasion, chemosis), ophthalmoplegia, retinal or vitreous hemorrhage, traumatic optic neuropathy, orbital fracture, retinal detachment, retinal tear and choroidal rupture. Final

\footnotetext{
*Address correspondence to this author at the Department of Ophthalmology, New York University School of Medicine, USA;

E-mail: jrperalta@gmail.com
}

visual acuity correlated to location of foreign body. $85 \%$ (11 of 13) of patients with anterior IoFBs retained final visual acuity greater than $20 / 40$, compared to only $30 \%$ ( 3 of 10 ) of patients with posterior IoFBs. Additionally, of the 3 patients who developed NLP vision, all had posterior IoFBs. It was deemed that management of each case was dependent on location of the projectile, and foreign bodies not readily surgically accessible may be left safely in place.

More recently, a study by Fulcher et al. expanded on the work by Finkelstein and colleagues [2]. In this slightly larger $(n=40)$ retrospective consecutive case series conducted over 10 years, foreign body composition was more varied. Projectiles were classified as metallic, non-metallic organic (wood) and non-metallic inorganic (glass, plastic, fiberglass, concrete). Associated ocular morbidities were slightly more serious than the previous study. Most common was perforating eye injury, occurring in $25 \%$ (10 of 40 ) of patients. Others included infection (orbital cellulitis, orbital abscess, cerebral abscess), ophthalmoplegia, ptosis, optic nerve trauma (pain with eye movement, optic neuropathy) and orbital fracture. $85 \%$ (34 of 40) of patients underwent surgical removal. All 6 cases that did not had posteriorly located IoFBs. In reviewing the surgical indications, the most important factors were foreign body location and composition. To aid in these situations, the following algorithm was developed (Fig. 2) [2-4].

It is first most important to complete a full systemic evaluation and to rule out an ophthalmologic emergency (i.e. ruptured globe). Empiric broad-spectrum antibiotics should be administered to all patients. Appropriate imaging can then be obtained to help identify the foreign body. Computed tomography is preferred as magnetic resonance imaging should be avoided until a metallic foreign body can be unequivocally ruled-out. All organic foreign bodies should be removed, although there is a paucity of data on those located in the posterior orbit. The approach to inorganic foreign bodies is more complex. Those that are located anteriorly and freely palpable should be removed. Non-palpable anterior, epibulbar and posterior foreign bodies can be managed conservatively given the risk of collateral damage during surgical extraction. Most retained metallic IoFBs are welltolerated and typically have minimal adverse visual prognosis 

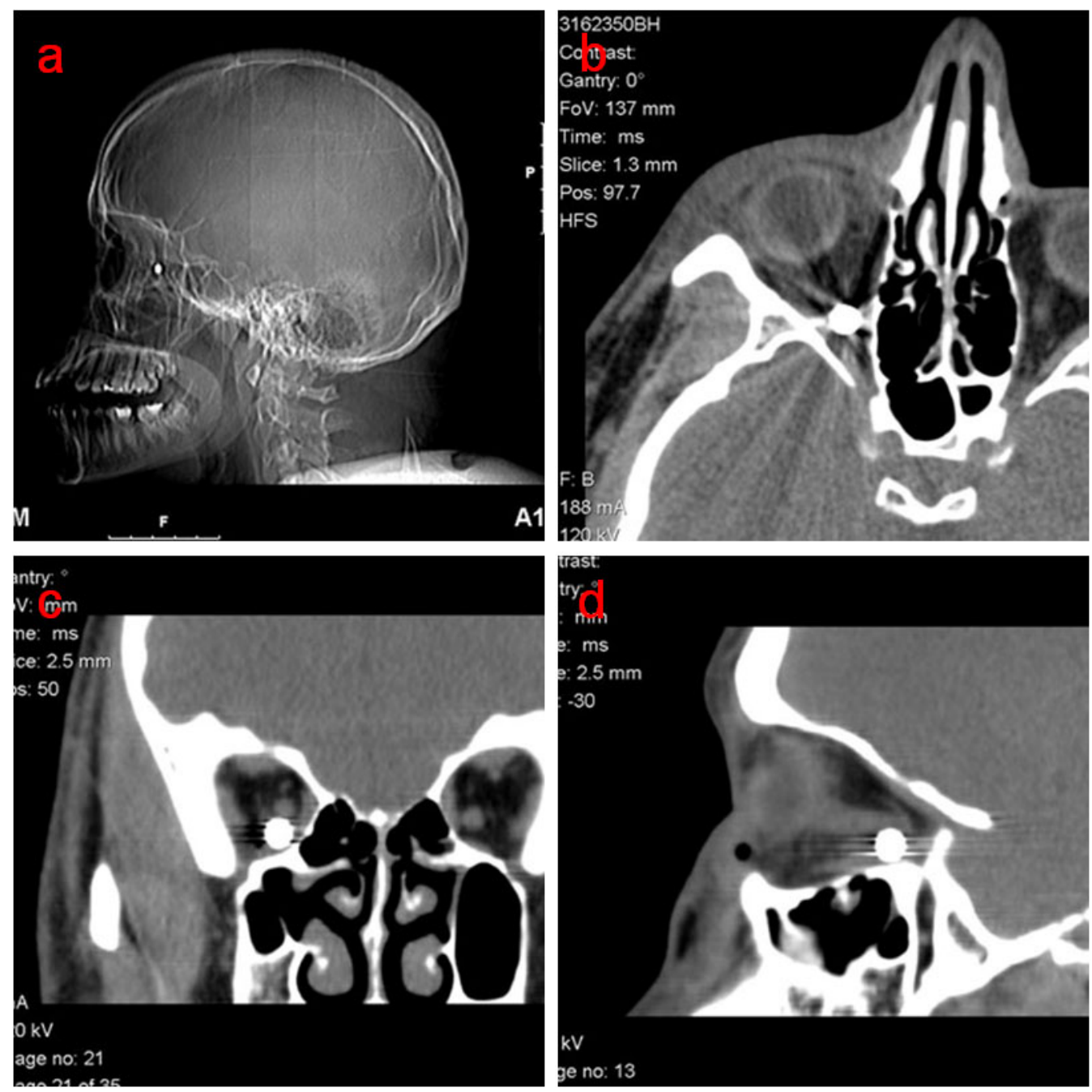

Fig. (1). (a) Computed tomography of the brain and orbits demonstrates a metallic intraorbital foreign body located deep in the orbit. Axial (b), coronal (c) and sagittal (d) sections further delineate the proximity of the foreign body to the optic nerve.

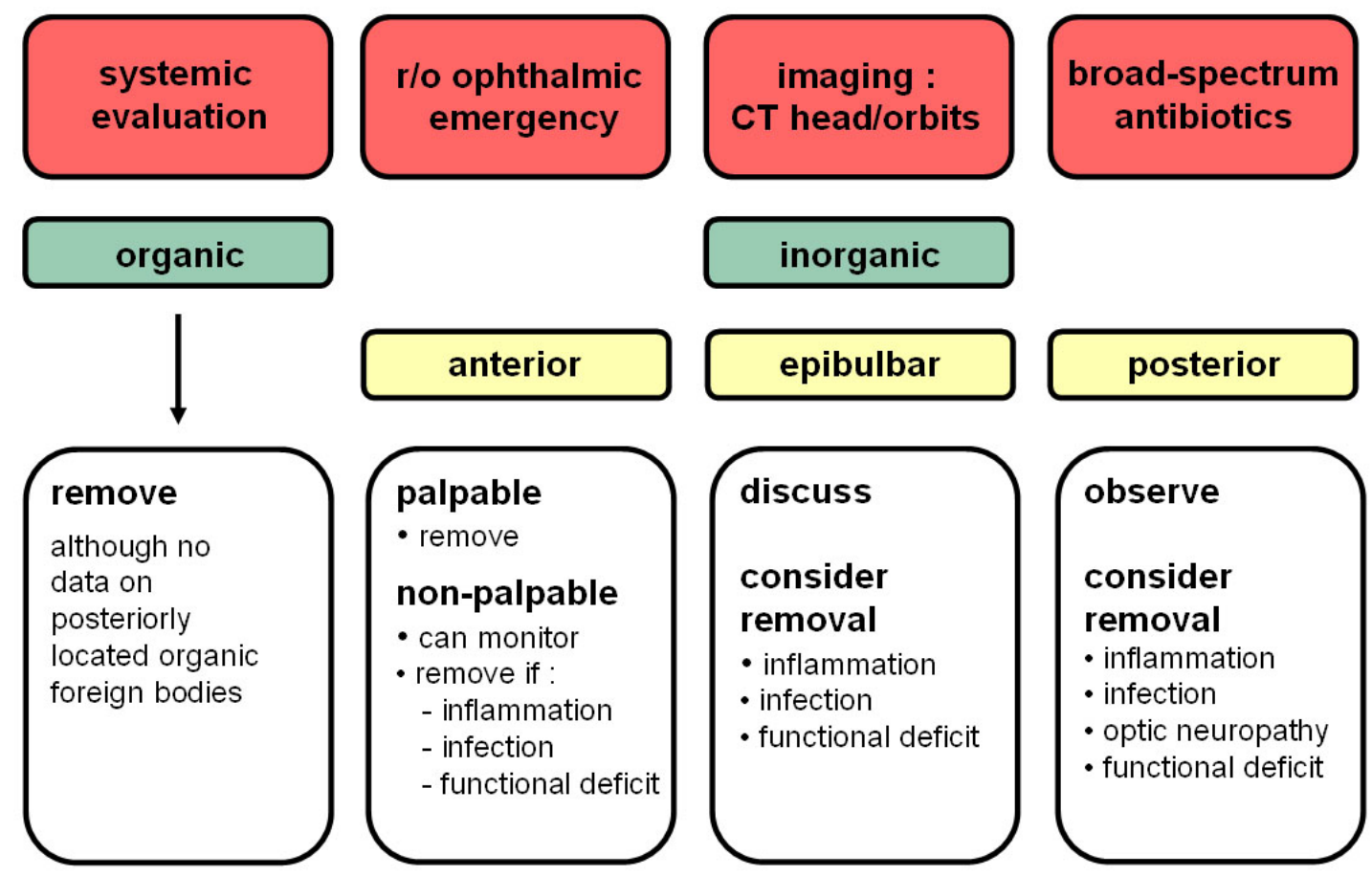

Fig. (2). Management algorithm for intraorbital foreign body. 
[3]. In cases with associated traumatic optic neuropathy, the use of corticosteroid therapy is still debated among physicians [5]. There is insufficient evidence to conclude that corticosteroid therapy provides benefit in cases of traumatic optic neuropathy; however, in this case report, the patient demonstrated excellent clinical response. Removal should be considered in cases associated with inflammation, infection or a functional deficit.

\section{CONCLUSIONS}

An inert, well-tolerated metallic foreign body located deep in the posterior orbit may be conservatively managed with observation and appropriate supportive care, thus avoid- ing potential iatrogenic injury to vital structures adjacent to the orbital apex.

\section{REFERENCES}

[1] Finklestein M, Legmann A, Rubin PA. Projectile metallic foreign bodies in the orbit. Ophthalmology 1997; 104: 96-103.

[2] Fulcher TP, McNab AA, Sullivan TJ. Clinical features and management of intraorbital foreign bodies. Ophthalmology 2002; 190: 494-500.

[3] Ho V, Wilson MW, Fleming JC, Haik BG. Retained intraorbital metallic foreign bodies. Ophthal Plast Reconstr Surg 2004; 20 232-6.

[4] Michon J, Liu D. Intraorbital foreign bodies. Semin Ophthalmol 1994; 9: 193-9.

[5] Yu-Wai-Man P, Griffiths PG. Steroids for traumatic optic neuropathy. Cochrane Database Syst Rev 2007; (4): CD006032. 